\title{
Equilibrium in Combinatorial Public Projects
}

\author{
Brendan Lucier ${ }^{1}$, Yaron Singer ${ }^{2 \star}$, Vasilis Syrgkanis ${ }^{3 \star \star}$, and Eva Tardos ${ }^{3 \star \star \star}$ \\ 1 Microsoft Research \\ brlucier@microsoft.com \\ 2 School of Engineering and Applied Sciences, Harvard University \\ yaron@seas. harvard.edu \\ 3 Dept. of Computer Science, Cornell University, Ithaca, NY, USA \\ vasilis@cs.cornell.edu, eva@cs.cornell.edu
}

\begin{abstract}
We study simple item bidding mechanisms for the combinatorial public project problem and explore their efficiency guarantees in various well-known solution concepts. We first study sequential mechanisms where each agent, in sequence, reports her bid for every item in a predefined order on the agents determined by the mechanism. We show that if agents' valuations are unit-demand any subgame perfect equilibrium of a sequential mechanism achieves the optimal social welfare. For the simultaneous bidding equivalent of the above auction we show that for any class of bidder valuations, all Strong Nash Equilibria achieve at least a $O(\log n)$ factor of the optimal social welfare. For Pure Nash Equilibria we show that the worst-case loss in efficiency is proportional to the number of agents. For public projects in which only one item is selected we show constructively that there always exists a Pure Nash Equilibrium that guarantees at least $\frac{1}{2}\left(1-\frac{1}{n}\right)$ of the optimum. We also show efficiency bounds for Correlated Equilibria and Bayes-Nash Equilibria, via the recent smooth mechanism framework [26].
\end{abstract}

\section{Introduction}

In recent years considerable attention has been devoted to the design and analysis of "simple" mechanisms: algorithms for strategic environments that yield provable guarantees yet are simple enough to run in practice. This trend is motivated by the realization that mechanisms which are implemented in practice and encourage participation cannot be arbitrarily complex. Since simplicity often comes at the price of lowered economic efficiency, the goal in analyzing simple mechanisms is to quantify this loss in comparison to some theoretical optimum.

Simple mechanisms have been explored thus far primarily in auction domains. Some examples include posted price mechanisms that approximate revenueoptimal auctions [5], and simultaneous single item auctions that have good social efficiency relative to the fully efficient combinatorial auctions $[6,1,13,10]$.

* Part of this work was done while the author was visiting Cornell University

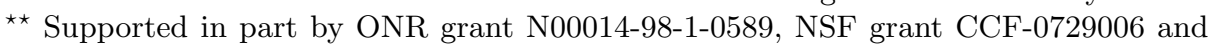
a Simons Graduate Fellowship.

*** Supported in part by NSF grants CCF-0910940 and CCF-1215994, ONR grant N00014-08-1-0031, a Yahoo! Research Alliance Grant, and a Google Research Grant. 
In contrast to auctions where agents compete for allocated resources, public projects require agents to coordinate on resources that are collectively allocated. This is captured in the combinatorial public projects model introduced in [21], where there are multiple resources (items), each agent has a combinatorial valuation function on subsets of the items, and the goal is to select some fixed number of items that maximizes the sum of the agents' valuations. This problem provides the first evidence of the computational hardness of truthful implementation: for agents with nondecreasing submodular valuations, there are constant-factor approximation algorithms when agents' valuations are known, but there is no computationally efficient truthful mechanism that can obtain a reasonable approximation under standard complexity-theoretic assumptions [21].

As a canonically hard mechanism design problem, there has been an ongoing investigation of mechanisms for combinatorial public projects under various valuation classes and solution concepts $[3,12,8,14,7]$. When considering simple mechanisms, the item-bidding with first-prices mechanism, which is the analogue of those used in combinatorial auctions $[6,1,13]$, is arguably the simplest non-trivial mechanism for combinatorial public projects: the mechanism asks each agent, simultaneously, to report her valuation separately for each item, then chooses the $k$ items whose sum of reported valuations is maximal and charges agents first prices, i.e. each agent pays her reported valuation for every item selected by the mechanism. Despite its appealing simplicity, it turns out that achieving desirable efficiency guarantees at equilibrium is not trivial in this mechanism. In evidence, consider an instance with $n$ agents and 3 items $A, B, C$, in which a single item is to be selected and agents valuations are as those summarized in the following $n \times 3$ matrix:

$$
\left[v_{i j}\right]=\left[\begin{array}{ccc}
n-1 & 1 & 0 \\
\vdots & \vdots & \vdots \\
n-1 & 1 & 0 \\
0 & 0 & n-1
\end{array}\right]
$$

In this instance all the agents except agent $n$ have a valuation $v_{i A}=n-1$ for $A, v_{i B}=1$ for item $B$, and $v_{i C}=0$ for item $C$, and agent $n$ has a valuation of 0 for items $A$ and $B$ and a valuation of $n-1$ for project $C$. Obviously the optimal outcome is for $A$ to be chosen leading to a social welfare of $(n-1)^{2}$. However, there exists a Nash Equilibrium where project $B$ is selected: if all agents except agent $n$ bid 1 for $B$ and 0 for $A$ and agent $n$ bids truthfully, then in this profile the bids on $B$ total to $(n-1)$ (assuming tie-breaking is chosen in favor of $B$ ) and $B$ is selected. To see that this an equilibrium, note that for any agent except $n$ the only two ways to alter the allocation is either by only reducing the current bid for $B$ to 0 and letting $C$ be selected, or reducing the bid for $B$ to 0 and increasing his bid on $A$ to $n-1$. For both deviations the utility (valuation minus payment) would be 0 , which is exactly what the agent is currently getting.

The above example shows that even when agents have very simple valuation functions (unit-demand), the price of anarchy - the ratio between the optimal solution and that achievable in equilibrium - can be as bad as linear in the num- 
ber of agents in the system. It may therefore seem like simple mechanisms for combinatorial public projects are of little interest to anyone interested in reasonable efficiency guarantees. However, a more careful observation at the above example leaves some hope. The major difficulty in designing simple and efficient mechanisms for combinatorial public projects is the inability of the bidders to coordinate on the right equilibrium. Thus, to achieve worst case efficiency guarantees, either the mechanism should allow for agents to signal among each other, or the solution concept should allow for such signaling. Alternatively, an optimistic designer could be interested in best-case guarantees by studying the existence of good equilibria (Price of Stability), rather than that any equilibrium will be good, since the designer himself could somehow signal which equilibrium should be chosen. In this work we address all these three different routes. We study both sequential and simultaneous bidding mechanisms. In sequential bidding, the mechanism determines some order in which agents place their bids, while in the simultaneous case all agents bid simultaneously.

\subsection{Main Results}

To understand outcome quality in our item bidding mechanisms, we consider a number of standard solution concepts.

Sequential Item-Bidding Mechanism. We start by analyzing a sequential version of the item-bidding first-price mechanism, where the agents are asked sequentially (in an arbitrary order) to report their willingness-to-pay separately for each item. We focus on the subgame-perfect equilibria of this extensive form game, which is the most well-established concept for sequential games. We show that when bidders have unit-demand valuations (i.e. their valuation when a set $S$ of items is chosen is their maximum valued item in the set) then every subgame-perfect equilibrium achieves optimal social welfare. We reiterate that in the simultaneous mechanism the inefficiency can grow linearly with the number of players even for unit-demand valuations, as illustrated in the example given above, rendering the design of good mechanisms for such valuations a non-trivial task. The intuition behind our result is that the sequentiality of the moves allows the agents to signal their preferences and to coordinate on a specific equilibrium by pre-committing on their declared valuations.

Strong-Nash Equilibria of the Simultaneous Item-Bidding Mechanism. Subsequently we analyze the quality of Strong Nash Equilibria (equilibria that are stable under coalitional deviations) of the simultaneous item-bidding mechanism, where all agents are asked to simultaneously submit their willingness-to-pay separately for each item. Our simple mechanism for the public project problem can be thought of as a coordination game, where agents need to coordinate on their best set of items. In this context the Strong Nash Equilibrium is a very natural solution concept. We show that the loss in efficiency (the strong price of anarchy) is no more than $O(\log n)$. Essentially, Strong Nash Equilibria alleviate the coordination problem inherent in public project auctions by allowing the agents to 
coallitionally deviate if they found themselves stuck at a bad equilibrium where no agent unilaterally could affect the set of chosen items.

Nash Equilibria of the Simultaneous Item-Bidding Mechanism. Next we consider the quality of Pure Nash Equilibria of the simultaneous auction, and show that the worst-case loss in efficiency is proportional to $n$, the number of agents ${ }^{4}$. Our upper bound requires no assumption on bidders' valuations. Note that while the $n$ bound on the price of anarchy seems weak, it is better than any deterministic truthful mechanisms: [21] shows that computationally efficient deterministic dominant strategy mechanisms cannot do better than $\sqrt{m}$ (where $m$ is the number of resources). For the special case of unit-demand agents (whose valuation for a set of items is the value of the best item selected) we give an improved bound of $n / k$, where $k$ is the number of items that need to be chosen.

The high inefficiency of the worst pure nash equilibrium is due to the fact that certain bad equilibria survive due to the lack of good unilateral deviations. However, such equilibria tend to be unreasonable and unnatural. Thus it is interesting to study the existence of good equilibria of the auction. For the case when one item is to be chosen we show constructively that the best Pure Nash Equilibrium is guaranteed to obtain at least a $\frac{1}{2}\left(1-\frac{1}{n}\right)$ fraction of the optimal welfare.

Learning Behavior and Incomplete Information. The equilibrium analysis so far assumed that agents will reach a stable solution of the bidding game, i.e., an equilibrium. We also explore the quality of solution achieved in a repeated version of the simultaneous game under the weaker assumption that all agents employ no-regret learning strategies. If all agents use no-regret learning strategies [2], than the resulting outcome distribution is a coarse correlated equilibrium of the game. We show that the loss in efficiency of any coarse correlated equilibrium is no more than $2 \cdot n \cdot k$ for arbitrary valuations, $\frac{2 e}{e-1} n$ for fractionally subadditive valuations and $\frac{e}{e-1} n / k$ for unit-demand valuations. The latter bounds are given via the smooth-mechanism framework [26] and thereby also carry over to the set of Bayes-Nash equilibria of the incomplete information setting, where valuations are private and drawn from commonly known distributions.

\section{$1.2 \quad$ Related Work}

There is a long recent literature on combinatorial public projects that mainly tries to find truthful mechanisms with good efficiency guarantees $[21,24,3,8]$. Specifically, as mentioned above, in [21] it is shown that under standard assumptions, no tractable truthful mechanism can achieve an approximation factor better than $\sqrt{m}$ for agents with nondecreasing submodular valuations.

There has been a long line of research on quantifying inefficiency of equilibria starting from [15] who introduced the notion of the price of anarchy. Several

\footnotetext{
${ }^{4}$ We also show that this bound is essentially tight, by giving a lower bound of $n-1$ on the price of anarchy.
} 
recent papers have studied the efficiency of simple mechanisms. A series of papers, Christodoulou, Kovacs and Schapira [6], Bhawalkar and Roughgarden [1] Hassidim, Kaplan, Mansour, Nisan [13], and Feldman et al. [10], studied the inefficiency of Bayes-Nash equilibria of non-truthful combinatorial auctions that are based on running simultaneous separate single-item auctions. Lucier and Borodin studied Bayes-Nash Equilibria of non-truthful auctions based on greedy allocation algorithms [16]. Paes Leme and Tardos [20], Lucier and Paes Leme [17] and Caragiannis et al. [4] studied the ineffficiency of Bayes-Nash equilibria of the generalized second price auction. Roughgarden [22] showed that many price of anarchy bounds carry over to imply bounds also for learning outcomes. Roughgarden [23] and Syrgkanis [25] showed that such bounds also extend to bound the inefficiency of games of incomplete information. Recently, in [26] we give a more specialized framework for the case of non-truthful mechanisms in settings with quasi-linear preferences, showing how to capture several of the previous results. In this work we show that our upper bounds for coarse correlated and Bayes-Nash equilibria of the simultaneous auction fall into the framework of $[26]$.

The quality of subgame-perfect equilibria of sequential versions of simultaneous games, was introduced in [19] and has been applied to cost-sharing games, cut and consensus games, load balancing games. Our result on the sequential item-bidding mechanism is of similar flavor to this line of work and gives another interesting application of the latter approach.

\section{Model and Notation}

In combinatorial public projects there is a set of $m$ items and $n$ agents. Each agent $i \in[n]$ has a valuation function $v_{i}: 2^{[m]} \rightarrow \mathbb{R}_{\geq 0}$ for each set of chosen items. Given some fixed parameter $k$, the goal of the designer is to select a set $S$ of size $k$ that maximizes the total valuation of the agents $V(S)=\sum_{i} v_{i}(S)$. Since agents' valuations are considered private information, the mechanism enforces payments to help achieve good equilibria. For a profile of agents' bids $b$, when the selected subset by the mechanism is $S$ and the payments profile is $p=\left(p_{1}, p_{2}, \ldots, p_{n}\right)$, the utility of an agent $i$ denoted $u_{i}(b)$ is $v_{i}(S)-p_{i}$.

Valuation Classes. In some cases we state results over arbitrary valuation classes, and in others our results are stated for valuation classes that have particular combinatorial structure. A valuation $v$ is additive if $v(S)=\sum_{j \in S} v(\{j\})$ for all $S \subseteq[m]$. A valuation $v$ is (nondecreasing) submodular if it has a decreasing marginal utilities property: $v(S)-v(S \cup\{j\}) \geq v(T)-v(T \cup\{j\})$ for $S \subseteq T$ and all $j \in[m]$. A valuation $v$ is unit-demand if $v(S)=\max _{j \in S} v(\{j\})$. It is easy to see that every unit-demand valuation is submodular. ${ }^{5}$

\footnotetext{
${ }^{5}$ In our case, when agents have submodular valuations, the algorithmic problem becomes that of maximizing a submodular function under a cardinality constraint for which there is a computationally efficient greedy algorithm that is well-known to be
} 
A generalization of submodular valuations which we will use in this work is that of fractionally subadditive (or XOS) valuations (see [9]): a valuation $v$ is fractionally subadditive if and only if there exist a set of additive valuations $\left(v^{\ell}\right)_{\ell \in \mathcal{L}}$ such that $v(S)=\max _{\ell \in \mathcal{L}} v^{\ell}(S)$.

The First-Price Item-Bidding Mechanism. We consider the following simple item-bidding mechanism. Each agent $i \in[n]$ submits a bid $b_{i j}$ for each item $j \in[m]$. For an item $j \in[m]$ let $B_{j}=\sum_{i} b_{i j}$ be the total bid placed on $j$. The mechanism chooses the $k$ items with the highest total bids. For profile $b$ let $S(b)$ be the chosen set. Each agent is charged her bids for the chosen items: $p_{i}=\sum_{j \in S(b)} b_{i j}$. We consider two variants: in the simultaneous mechanism, all agents submit their bids simultaneously. In the sequential mechanism, the agents submit their bids sequentially in some order, with each agent seeing the bids of those who came before. We define solution concepts for both mechanisms.

Solution Concepts for Simultaneous Games. We now define the main solution concepts that we will use in the context of the simultaneous move mechanism. A Pure Nash Equilibrium (PNE) is a set of bids $\left(b_{i j}\right)_{i \in[n], j \in[m]}$ such that, for each agent $i$, there is no bid vector $\boldsymbol{b}_{i}^{\prime}$ such that

$$
u_{i}\left(\boldsymbol{b}_{i}^{\prime}, \boldsymbol{b}_{-i}\right)>u_{i}(\boldsymbol{b}) .
$$

If we allow the agents to make coalitional deviations then the appropriate equilibrium concept is Strong Nash Equilibrium (SNE). A set of bids $\left(b_{i j}\right)_{i \in[n], j \in[m]}$ constitutes a SNE if, for each set of agents $S \subseteq[n]$, there is no bid vector $\boldsymbol{b}_{S}^{\prime}=\left(\boldsymbol{b}_{i}^{\prime}\right)_{i \in S}$ such that

$$
\forall i \in S: u_{i}\left(\boldsymbol{b}_{S}^{\prime}, \boldsymbol{b}_{-S}\right)>u_{i}(\boldsymbol{b}) .
$$

A relaxed notion of equilibrium corresponds to no-regret learning outcomes (due to space limitations see [2] for a survey). It is known that such learning outcomes correspond to Coarse Correlated Equilibria of a game. A (possibly correlated) distribution on bids $\boldsymbol{b} \sim D$ is a Coarse Correlated Equilibrium if, for every agent $i$ and for every bid $\boldsymbol{b}_{i}^{\prime}$,

$$
\mathbb{E}_{\boldsymbol{b} \sim \mathcal{D}}\left[u_{i}(\boldsymbol{b})\right] \geq \mathbb{E}_{\boldsymbol{b} \sim \mathcal{D}}\left[u_{i}\left(\boldsymbol{b}_{i}^{\prime}, \boldsymbol{b}_{-i}\right)\right]
$$

that is, no agent $i$ can improve his expected utility by unilaterally deviating.

All of the above equilibrium notions implicitly assume a full-information model, where agent valuations are commonly known. In the alternative model of incomplete information, the valuation profile $\boldsymbol{v}$ is drawn from distribution $\boldsymbol{F}$, where this distribution is common knowledge. In the Item-Bidding Mechanism under incomplete information, each agent's strategy is a function $b_{i}\left(v_{i}\right)$ that outputs an agent's bids given her realized valuation. A Bayes-Nash Equilibrium of this game is a profile of strategies $b(v)=\left(b_{i}\left(v_{i}\right)\right)_{i \in N}$ such that

$$
\forall i \in N, \forall v_{i}: E_{\mathbf{v}_{-\mathbf{i}} \mid v_{i}}\left[u_{i}\left(b_{i}\left(v_{i}\right), b_{-i}\left(\mathbf{v}_{-\mathbf{i}}\right)\right)\right] \geq E_{\mathbf{v}_{-\mathbf{i}} \mid v_{i}}\left[u_{i}\left(b_{i}^{\prime}\left(v_{i}\right), b_{-i}\left(\mathbf{v}_{-\mathbf{i}}\right)\right]\right.
$$

within a factor of $1-1 / e$ of the optimum (assuming that agents comply with the protocol and reveal their true valuations) [18]. 
The Sequential Item-Bidding Mechanism. In the sequential item-bidding mechanism, the agents are ordered under some arbitrary but commonly-known predefined sequence. Each agent is asked sequentially, in this order, to report a bid $b_{i j}$ for each item $j \in[m]$. After all the agents have reported their bids, the mechanism chooses the set of $k$ items with the highest total bid $B_{j}=\sum_{i} b_{i j}$ and charges each agent her bid on each selected item. In this sequential game the strategy of each agent is not simply a set of bids $b_{i j}$ for each item $j \in[m]$ but rather it is a contingency of plans describing how the agent will bid conditioned on the history of play up to her turn. If we denote with $h_{i}$ the history of play (i.e. reported bids) up to agent $i$ then the strategy of an agent is a set of functions $b_{i j}\left(h_{i}\right)$ that maps each history to a bid vector.

Subgame-Perfect Equilibrium. A natural solution concept for sequential games is Subgame-Perfect Equilibrium, a refinement of the Nash Equilibrium. A profile of strategies is a subgame-perfect equilibrium if it constitutes an equilibrium on any subgame induced for any possible history of play. Note that this definition restricts the behavior of agents outside the equilibrium path, ruling out non-viable threats (for detailed discussion of subgame-perfection see [11]).

Measure of Efficiency. For each equilibrium notion above, we can measure worst-case efficiency by way of the price of anarchy. For a given equilibrium concept, the corresponding price of anarchy is the ratio between the minimum expected welfare of any equilibrium (with expectation over randomness in the strategies and/or realizations of bidders' valuations) and the expected optimal social welfare (over randomness in the bidders' valuations).

\section{Sequential Item-Bidding Mechanism}

We begin by considering outcomes of the sequential item-bidding mechanism at subgame-perfect equilibrium. We will focus on the case that agents have unitdemand valuations, where we find that the price of anarchy is 1 . That is, the agents always select an optimal outcome. Note that this is in contrast to the example discussed in the introduction which shows that the price of anarchy (of Nash equilibrium) for the simultaneous item-bidding mechanism can be as large as $n-1$.

Theorem 1. For unit-demand valuations and any $k \geq 1$, the unique subgame perfect equilibrium of the sequential item-bidding mechanism selects a welfareoptimal outcome. Moreover, at this equilibrium each agent bids on a single item.

Proof. For any value profile $V$, let $O P T(V)=\operatorname{argmax}_{S:|S|=k}\left\{\sum_{i} v_{i}(S)\right\}$, breaking ties arbitrarily. Throughout the proof we will think of a bid vector $\mathbf{b}_{i}$ as an additive valuation function $b_{i}$, so that (in particular) $O P T(\mathbf{b})$ is the outcome selected by the mechanism when agents submit bids $\mathbf{b}$. We will also write $V(S)=\sum_{i} v_{i}(S)$ for the social welfare of an outcome $S$ under valuations $V$. 
Let $\mathbf{b}$ be an arbitrary bid profile, and for each $i$ consider the valuation profile $\mathbf{b}^{(i)}=\left(b_{1}, b_{2}, \ldots, b_{i}, v_{i+1}, \ldots, v_{n}\right)$. Note $\mathbf{b}^{(0)}=V$ and $\mathbf{b}^{(n)}=\mathbf{b}$. For each $i \geq 1$, consider the subgame that occurs just before agent $i$ is about to bid, after agents 1 through $i-1$ bid according to $\mathbf{b}$. We will show by backward induction that the unique equilibrium of this subgame selects outcome $O P T\left(\mathbf{b}^{(i-1)}\right)$, and each agent bids on at most one item in this equilibrium. Taking $i=1$ will then prove our theorem, since then $\operatorname{OPT}\left(\mathbf{b}^{(0)}\right)=O P T(V)$ is the outcome of the mechanism.

The base case $i=n+1$ is trivial, since by definition the mechanism selects outcome $\operatorname{OPT}(\mathbf{b})=\operatorname{OPT}\left(\mathbf{b}^{(n)}\right)$. For $i \leq n$, we know by induction that, for any bid $b_{i}$ made by agent $i$, the mechanism returns $\operatorname{OPT}\left(b_{i}, \mathbf{b}_{-i}^{(i)}\right)$. We must show that, for the utility-maximizing bid $b_{i}$ for agent $i, \operatorname{OPT}\left(b_{i}, \mathbf{b}_{-i}^{(i)}\right)=\operatorname{OPT}\left(\mathbf{b}^{(i-1)}\right)$.

One potential strategy for agent $i$ is to bid nothing (i.e., the zero bid $\mathbf{0}$ ), obtaining utility $v_{i}\left(\operatorname{OPT}\left(\mathbf{0}, \mathbf{b}_{-i}^{(i-1)}\right)\right)$. Since $v_{i}$ is unit-demand, the only way to obtain higher utility is to choose some $j$ with $v_{i}(j)>v_{i}\left(O P T\left(\mathbf{0}, \mathbf{b}_{-i}^{(i-1)}\right)\right)$ and bid some (minimal) $b_{i}$ so that $j \in O P T\left(b_{i}, \mathbf{b}_{-i}^{(i)}\right)$. Note that this $b_{i}$ will place a positive bid only on item $j$; let $x_{j}$ be the minimal value such that $j \in O P T\left(b_{i}, \mathbf{b}_{-i}^{(i)}\right)$ when $b_{i}(j)=x_{j}$. If agent $i$ makes this minimal bid for $j$, he obtains utility $v_{i}(j)-x_{j}$.

We have argued that agent $i$ maximizes utility by bidding on at most one item, so (by induction) each agent's bid at equilibrium is unit-demand.

Recalling the definition of $x_{j}$, let $S_{j} \ni j$ be the set selected by the mechanism when $i$ bids $x_{j}$ on $j$. Since all valuations in $\mathbf{b}_{-i}^{(i-1)}$ are unit demand, we have

$$
x_{j}=\mathbf{b}_{-i}^{(i-1)}\left(\operatorname{OPT}\left(\mathbf{0}, \mathbf{b}_{-i}^{(i-1)}\right)\right)-\mathbf{b}_{-i}^{(i-1)}\left(S_{j}\right) .
$$

We now consider two cases. First, suppose $i$ maximizes utility by bidding nothing, so $b_{i}=\mathbf{0}$. Then $O P T\left(\mathbf{b}^{(i)}\right)=\operatorname{OPT}\left(\mathbf{0}, \mathbf{b}_{-i}^{(i-1)}\right)$. We will show $O P T\left(\mathbf{b}^{(i-1)}\right)=$ $\operatorname{OPT}\left(\mathbf{0}, \mathbf{b}_{-i}^{(i-1)}\right)$, by showing that each set $S_{j}$ achieves lower social welfare than $\operatorname{OPT}\left(\mathbf{0}, \mathbf{b}_{-i}^{(i-1)}\right)$ under profile $\mathbf{b}^{(i-1)}$. (This suffices because, as argued above, $\operatorname{OPT}\left(\mathbf{b}^{(i-1)}\right)$ must be either $\operatorname{OPT}\left(\mathbf{0}, \mathbf{b}_{-i}^{(i-1)}\right)$ or $S_{j}$ for some item $j$, since $v_{i}$ is unit-demand). Pick any $j \notin O P T\left(\mathbf{0}, \mathbf{b}_{-i}^{(i-1)}\right)$. Since $b_{i}$ is utility-maximal, $v_{i}\left(O P T\left(\mathbf{0}, \mathbf{b}_{-i}^{(i-1)}\right)\right) \geq v_{i}(j)-x_{j}$. Substituting (4) and rearranging, we get that $\mathbf{b}^{(i-1)}\left(O P T\left(\mathbf{0}, \mathbf{b}_{-i}^{(i-1)}\right)\right) \geq v_{i}(j)+\mathbf{b}_{-i}^{(i-1)}\left(S_{j}\right)=\mathbf{b}^{(i-1)}\left(S_{j}\right)$ and hence we conclude $\mathbf{b}^{(i-1)}\left(O P T\left(\mathbf{0}, \mathbf{b}_{-i}^{(i-1)}\right)\right) \geq \mathbf{b}^{(i-1)}\left(S_{j}\right)$ as required.

Next suppose that $i$ maximizes his utility by choosing $b_{i}$ to be a bid of $x_{j}$ on item $j$. We will show that $\operatorname{OPT}\left(\mathbf{b}^{(i-1)}\right)=S_{j}$, by showing that neither $O P T\left(\mathbf{0}, \mathbf{b}_{-i}^{(i-1)}\right)$ nor $S_{j^{\prime}}$ for $j^{\prime} \neq j$ can achieve higher welfare under valuation profile $\mathbf{b}^{(i-1)}$. Since $i$ maximized utility by bidding on $j$, we have $v_{i}\left(\operatorname{OPT}\left(\mathbf{0}, \mathbf{b}_{-i}^{(i-1)}\right)\right) \leq$ $v_{i}(j)-x_{j}$ and $v_{i}(j)-x_{j} \geq v_{i}\left(j^{\prime}\right)-x_{j^{\prime}}$ for all $j^{\prime} \neq j$. Rearranging these inequalities implies $\mathbf{b}^{(i-1)}\left(\operatorname{OPT}\left(\mathbf{0}, \mathbf{b}_{-i}^{(i-1)}\right)\right) \leq \mathbf{b}^{(i-1)}\left(S_{j}\right)$ and $\mathbf{b}^{(i-1)}\left(S_{j^{\prime}}\right) \leq \mathbf{b}^{(i-1)}\left(S_{j}\right)$ for all $j^{\prime} \neq j$, and hence $O P T\left(\mathbf{b}^{(i-1)}\right)=S_{j}$ as required.

In either case, we have that the equilibrium at this subgame selects outcome $O P T\left(\mathbf{b}^{(i-1)}\right)$. The theorem now follows by induction. 


\section{Strong Nash Equilibrium of the Simultaneous Item-Bidding Mechanism}

In this section we give efficiency bounds for strong Nash equilibria of the simultaneous item-bidding mechanism. A state of a game is a strong Nash equilibrium (SNE) if there is no coalition of agents that can each individually benefit by deviating as a group. Despite being a strong requirement, SNE is a natural solution concept in public projects as allocations of resources are collectively shared by agents.

Theorem 2. Any Strong Nash Equilibrium of the first-price item bidding mechanism has efficiency at least $\log (n)$ of the optimal.

Proof. Let $B_{i}(A)$ be the sum of bids of agent $i$ for set $A$. Let $S$ be the set that is selected at a strong nash equilibrium and $O P T$ be the optimal set.

First we show that at any Strong Nash Equilibrium all the chosen projects receive the same $B_{j}$, i.e. $\forall j \in S: B_{j}=p$. Suppose that some chosen project has $B_{j}>p$. Then a agent $i$ who is bidding positively on this project could just decrease his bid by some $\epsilon$. The selected set would remain unchanged and agent $i$ would be paying $\epsilon$ less than before. Hence, his utility would increase.

Now, suppose that all the agents deviate to bidding some small $\epsilon$ only on the optimal set $O P T$. The definition of a strong Nash equilibrium states that there exists an agent that doesn't prefer the utility at the deviation. W.l.o.g. rearrange the agents such that it is agent 1 ; then $v_{1}(S)-B_{1}(S) \geq v_{1}(O P T)$. Now suppose that the agents $\{2, \ldots, n\}$ deviate to bidding each $\frac{p}{n-1}$ on each item in OPT. By definition of SNE there exists an agent (w.l.o.g., agent 2) that doesn't prefer this deviation; that is, $v_{2}(S)-B_{2}(S) \geq v_{2}(O P T)-\frac{k p}{n-1}$. By similar reasoning we can reorder the agents such that, for each $i$,

$$
v_{i}(S)-B_{i}(S) \geq v_{i}(O P T)-\frac{k p}{n-i+1}
$$

Summing all the above inequalities we get:

$$
\begin{aligned}
V(S)-\sum_{i} B_{i}(S) & \geq V(O P T)-k p \sum_{i=1}^{n} \frac{1}{n-i+1} \Longrightarrow \\
V(S)-k p & \geq V(O P T)-k p \log (n)
\end{aligned}
$$

Since $k p<V(S)$ we get that $V(S) \geq \frac{1}{\log (n)} V(O P T)$.

The above result gives a reasonable bound on the efficiency loss in such equilibria. Regarding existence of equilibrium, the non-existence for unit-demand agents when choosing two items and of PNE in Section 5 applies here as well, since SNE is a stronger solution concept. 


\section{Pure Nash Equilibria of the Simultaneous Item-Bidding Mechanism}

We now examine the efficiency and existence of Pure Nash Equilibria for the simultaneous item-bidding mechanism. For brevity we defer some proofs to the appendix. In [21] the $\sqrt{m}$ lower bound on truthful mechanisms applies to two agents with submodular valuations, and thus grows with the number of resources in the problem. In contrast, we show here that for the item bidding mechanism the loss in efficiency at any Pure Nash Equilibrium (whenever it exists) and for any type of bidder valuations is at most proportional to the number of agents.

Theorem 3. Any PNE of the item bidding mechanism has $P o A \leq n$.

Proof. As in the proof of Theorem 2 it is easy to see that at any Pure Nash Equilibrium all the chosen items receive the same $B_{j}$, i.e. $\forall j \in S: B_{j}=p$.

Let $b$ be a Nash Equilibrium and $S$ the chosen set. Let $O P T$ be the optimal set of items for the true valuations of the agents. Each agent $i$ could change the chosen set to $O P T$ by bidding $p+\epsilon$ on every item $j \in O P T$. Since we are at a Nash Equilibrium this deviation wouldn't be profitable:

$$
v_{i}(S)-\sum_{j \in S} b_{i j} \geq v_{i}(O P T)-k p
$$

Summing over all agents and using the fact that $\sum_{i \in[n]} \sum_{j \in S} b_{i j}=k \cdot p$ we get:

$$
V(S)-k \cdot p \geq V(O P T)-n \cdot k \cdot p
$$

Due to individual rationality no agent is paying above his total value. Hence, $k \cdot p \leq V(S)$. Thus: $n V(S) \geq V(O P T)$.

As shown in the Introduction, even when $k=1$ the PoA of unit-demand agents can be as bad as $n-1$, implying that our PoA upper bound is nearly tight. Note that when $k=1$ unit-demand and additive valuations coincide. Hence, our example proves that the PoA bound is tight even for additive agents.

Theorem 4. For unit-demand agents the PoA of the item bidding mechanism can be at least $n-1$, even when choosing a single item $(k=1)$.

Price of Stability. We now investigate existence of good pure Nash equilibria.

Theorem 5. There always exists a pure Nash equilibrium of the item bidding mechanism when $k=1$ and arbitrary number of agents. Moreover, it achieves at least $\frac{1}{2}\left(1-\frac{1}{n}\right)$ of the optimal social welfare.

Proof. For a set of agents $S$ and an item $j$ let: $V_{j}(S)=\sum_{i \in S} v_{i j}$. Moreover, let $a_{j, j^{\prime}}^{S}=V_{j}(S)-V_{j^{\prime}}(S)$. Let $a_{A, B}^{S^{*}}=\max _{S \subset N} \max _{j \in M} \max _{j^{\prime} \in M-j} a_{j, j^{\prime}}^{S}$, that is among all possible quantities $a_{j, j^{\prime}}^{S}, a_{A, B}^{S^{*}}$ is the maximum one. Observe that in the above maximum we take maximum only among sets that are strict subsets of $N$. In other words $S^{*} \subset N$ and $N-S^{*} \neq \emptyset$. The reason is that we need at least one agent to price set the "winners". We claim that the following outcome is an equilibrium: 
- $\forall i \in S^{*}: b_{i}(A)=v_{i A}-v_{i B}$ and $\forall j \neq A: b_{i}(j)=0$

$-\forall i \notin S^{*}: b_{i}(B)=v_{i B}-v_{i A}+\frac{a_{A, B}^{S *}-a_{B, A}^{N-S^{*}}}{n}$ and $\forall j \neq B: b_{i}(j)=0$

We denote with $p=\sum_{i \in S^{*}} b_{i}(A)$. Notice that by the definition of the equilibrium $p=\sum_{i \in S^{*}} b_{i}(A)=V_{A}\left(S^{*}\right)-V_{B}\left(S^{*}\right)=a_{A, B}^{S^{*}}$. Moreover, $p=\sum_{i \notin S^{*}} b_{i}(B)=$ $V_{B}\left(N-S^{*}\right)-v_{A}\left(N-S^{*}\right)+a_{A, B}^{S *}-a_{B, A}^{N-S^{*}}=a_{A, B}^{S^{*}}$.

We first focus on a agent $i \in S^{*}$. We take cases on his possible deviations and show that none is profitable:

- Drop bid on $A$ and let $B$ win: To show this is not profitable we need to show that $v_{i A}-b_{i}(A) \geq v_{i B} \Leftrightarrow v_{i A}-v_{i B} \geq b_{i}(A)$. From the equilibrium definition this is satisfied with equality.

- Drop bid on $A$ and bid $p$ on an item $j \neq A, B$ to make it win: We need to show that $v_{i A}-b_{i}(A) \geq v_{i j}-p \Leftrightarrow v_{i A}-v_{i A}+v_{i B} \geq v_{i j}-a_{A, B}^{S^{*}} \Leftrightarrow a_{A, B}^{S^{*}} \geq$ $v_{i j}-v_{i B}=a_{j, B}^{\{i\}}$. Which holds by the maximality of $a_{A, B}^{S^{*}}$.

Now we focus on a agent $i \notin S^{*}$.

- Slightly increase his bid on $B$ to make $B$ win: We need to show that $v_{i A} \geq$ $v_{i B}-b_{i}(B) \Leftrightarrow b_{i}(B) \geq v_{i B}-v_{i A}$. By the maximality of $a_{A, B}^{S^{*}}$, we have that $a_{A, B}^{S^{*}} \geq a_{B, A}^{N-S^{*}}$. Hence, the inequality holds by the definition of equilibrium.

- Drop bid on $B$ and bid $p$ on item $j \neq A, B$ to make it win: We need to show that $v_{i A} \geq v_{i j}-p \Leftrightarrow v_{i A} \geq v_{i j}-a_{A, B}^{S^{*}} \Leftrightarrow a_{A, B}^{S^{*}} \geq v_{i j}-v_{i A}=a_{j, A}^{\{i\}}$, which holds by the maximality of $a_{A, B}^{S^{*}}$.

In fact, above we gave a specific equilibrium where the price setting agents split equally the excess $a_{A, B}^{S^{*}}-a_{B, A}^{N-S^{*}}$, one can easily see that any splitting of that excess among the price setting agents is an equilibrium.

Efficiency. For the equilibrium constructed above we know that:

$$
\forall j \neq j^{\prime}, S^{\prime} \subset N: V_{A}\left(S^{*}\right)-V_{B}\left(S^{*}\right) \geq V_{j}\left(S^{\prime}\right)-V_{j^{\prime}}\left(S^{\prime}\right)
$$

Let $j^{*}$ be the optimal item. Consider the above property for $j=j^{*}, j^{\prime}=A$, and $S^{\prime}=N-\left\{\arg \min _{i \in N} v_{i j^{*}}\right\}$. The condition gives us:

$$
V_{A}\left(S^{*}\right)-V_{B}\left(S^{*}\right) \geq V_{j^{*}}\left(S^{\prime}\right)-V_{A}\left(S^{\prime}\right)
$$

By the definition of $S^{\prime}$ we know that $V_{j^{*}}\left(S^{\prime}\right) \geq\left(1-\frac{1}{n}\right) V_{j^{*}}(N)$. In addition $V_{A}\left(S^{*}\right) \leq V_{A}(N)$ and $V_{A}\left(S^{\prime}\right) \leq V_{A}(N)$. Combining all the above together we get:

$$
2 V_{A}(N) \geq V_{A}\left(S^{*}\right)+V_{A}\left(S^{\prime}\right)-V_{B}\left(S^{*}\right) \geq V_{j^{*}}\left(S^{\prime}\right) \geq\left(1-\frac{1}{n}\right) V_{j^{*}}(N)
$$

From this point onwards, due to lack of space we defer all proofs to the full version of the paper. For the case of two agents we can show existence of an optimal equilibrium.

Theorem 6. For $k=1, n=2$ there exists an optimal equilibrium. 
Existence and Complexity. In contrast to case where one project is chosen, we show that even when $k=2$ there may not exist a PNE when agents valuations are additive.

Theorem 7. For additive agents and $k=2$ there may not be a PNE.

Regarding complexity, the computational hardness can be shown by reducing the problem of finding a Pure Nash Equilibrium to that of the well-studied problem of finding maximal coverage of a universe of elements.

Theorem 8. It is NP-hard to compute a Pure Nash Equilibrium of the item bidding mechanism even for two agents with coverage valuations.

\section{Smoothness of the Item-Bidding Mechanism}

In this section we study the efficiency achieved at learning outcomes and also when players have incomplete information about the valuations of the rest of the players. We defer proofs to the appendix. We give efficiency bounds by utilizing the recently proposed Smooth Mechanism framework [26]. For completeness, we present the basic definition of smoothness and the theorem that we will utilize.

Definition 1 (Syrgkanis, Tardos [26]). A mechanism is $(\lambda, \mu)$-smooth if for any valuation profile $v$, there exist strategies $b_{i}^{\prime}\left(b_{i}, v\right)$, such that for any strategies $\boldsymbol{b}_{-i}$ of the rest of the players:

$$
\sum_{i} u_{i}\left(b_{i}^{\prime}\left(b_{i}, v\right), \boldsymbol{b}_{-i}\right) \geq \lambda O P T(v)-\mu \sum_{i} P_{i}(\boldsymbol{b})
$$

where $O P T(v)$ is the optimal social welfare for valuation profile $v$, and $P_{i}(\boldsymbol{b})$ is the payment of player $i$ under bid profile $\boldsymbol{b}$.

Theorem 9 (Syrgkanis, Tardos [26]). If a mechanism $(\lambda, \mu)$-smooth then the efficiency at any correlated equilibrium of the complete information game and at any Bayes-Nash equilibrium of the incomplete information game where $v_{i}$ 's are drawn from commonly known independent distributions $F_{i}$, is at least $\frac{\lambda}{\max \{\mu, 1\}}$. If the deviations $b_{i}^{\prime}\left(b_{i}, v\right)$ in Definition 1 are independent of $b_{i}$ then the latter holds also at coarse-correlated equilibria.

We show that for any class of bidder valuations and for any $k$ the ItemBidding Mechanism is a $\left(\frac{1}{2}, n \cdot k\right)$-smooth mechanism, thereby implying a Price of Anarchy of at most $2 n k$ for the aforementioned solution concepts.

Theorem 10. For agents with arbitrary monotone valuations the Item-Bidding Mechanism is $\left(\frac{1}{2}, n \cdot k\right)$-smooth.

When the bidders are fractionally subadditive, we show that the Item-Bidding Mechanism is $\left(\frac{1}{2}\left(1-\frac{1}{e}\right), n\right)$-smooth, implying a Price of Anarchy of at most $\frac{2 e}{e-1} n$, independent of the number $k$ of projects to be chosen. 
Theorem 11. For agents with fractionally subadditive monotone valuations the Item-Bidding Mechanism is $\left(\frac{1}{2}\left(1-\frac{1}{e}\right), n\right)$-smooth.

When players are unit-demand we are able to show that the Item-Bidding Mechanism actually satisfies the semi-smoothness property of Lucier et al. [17] which is essentially the special case of Definition 1 where the deviating bids depend only on a players own valuation. Such a stronger property allows for the efficiency guarantee of theorem 9 to carry over to incomplete information settings where the bidder distributions are correlated. We show that the mechanism is $\left(1-e^{-1}, \frac{n}{k}\right)$ semi-smooth for the case of unit-demand bidders implying a Price of Anarchy bound of at most $\frac{e}{e-1} \frac{n}{k}$, which decreases as the number of chosen project increases (i.e. more players are satisfied by building more projects).

Theorem 12. When bidder valuations are unit-demand then the Item-Bidding Mechanism is $\left(1-e^{-1}, \frac{n}{k}\right)$ semi-smooth.

\section{Discussion}

The work presented in this paper is a first step towards the broader understanding of equilibria induced in combinatorial public projects. More generally, we explore the tools for mechanism design under solution concepts other than dominant strategy truthfulness.

While our bounds for pure Nash equilibria are nearly tight, better efficiency bounds may perhaps be achieved for the other solution concepts we explored in this work. It may be possible that sublogarithmic bounds can be shown for Strong Nash Equilibria, or that constant factor bounds may be achieveable by subgame perfect equilibria beyond the case of unit-demand bidders.

The simple mechanism for public projects we analyzed here is the item bidding mechanism with first prices. This is arguably the simplest non-trivial mechanism in this setting, and can be extended in multiple ways; similar allocation rules with second prices, or including constraints on the allocation rule, may lead to substantially different results than the ones presented here. In particular, we believe there is a simple mechanism where agents can reach an efficient equilibrium through natural dynamics for public projects, even in settings where no reasonable Maximal-In-Range mechanisms exist.

\section{References}

1. K. Bhawalkar and T. Roughgarden. Welfare Guarantees for Combinatorial Auctions with Item Bidding. SODA '11, 2011.

2. A. Blum and Y. Mansour. Learning, regret minimization, and equilibria. In N. Nisan, T. Roughgarden, E. Tardos, and V. Vazirani, editors, Algorithmic Game Theory, chapter 4, pages 4-30. Cambridge University Press, 2007.

3. D. Buchfuhrer, M. Schapira, and Y. Singer. Computation and incentives in combinatorial public projects. In ACM Conference on Electronic Commerce, pages 33-42, 2010. 
4. I. Caragiannis, C. Kaklamanis, P. Kanellopoulos, and M. Kyropoulou. On the Efficiency of Equilibria in Generalized Second Price Auctions. In EC, 2011.

5. S. Chawla, J. D. Hartline, D. L. Malec, and B. Sivan. Multi-parameter mechanism design and sequential posted pricing. In Proceedings of the 42nd ACM symposium on Theory of computing, STOC '10, pages 311-320, New York, NY, USA, 2010. ACM.

6. G. Christodoulou, A. Kovács, and M. Schapira. Bayesian combinatorial auctions. ICALP '10, pages 820-832, 2010.

7. S. Dobzinski. An impossibility result for truthful combinatorial auctions with submodular valuations. In STOC, pages 139-148, 2011.

8. S. Dughmi. A truthful randomized mechanism for combinatorial public projects via convex optimization. In ACM Conference on Electronic Commerce, pages 263-272, 2011.

9. U. Feige. On maximizing welfare when utility functions are subadditive. In $S T O C$, 2006.

10. M. Feldman, H. Fu, N. Gravin, and B. Lucier. Simultaneous auctions are (almost) efficient. In $S T O C, 2013$.

11. D. Fudenberg and J. Tirole. Game Theory. MIT Press, 1991.

12. A. Gupta, K. Ligett, F. McSherry, A. Roth, and K. Talwar. Differentially private combinatorial optimization. In SODA, pages 1106-1125, 2010.

13. A. Hassidim, H. Kaplan, Y. Mansour, and N. Nisan. Non-price equilibria in markets of discrete goods. In EC '11, page 295, New York, New York, USA, June 2011. ACM Press.

14. Z. Huang and S. Kannan. The exponential mechanism for social welfare: Private, truthful, and nearly optimal. In FOCS, pages 140-149, 2012.

15. E. Koutsoupias and C. H. Papadimitriou. Worst-case equilibria. In STACS, pages:404-413, 1999.

16. B. Lucier and A. Borodin. Price of anarchy for greedy auctions. SODA'10, pages 0-20, 2010.

17. B. Lucier and R. Leme. GSP auctions with correlated types. In EC '11, 2011.

18. G. L. Nemhauser, L. A. Wolsey, and M. L. Fisher. An analysis of approximations for maximizing submodular set functions ii. Math. Programming Study 8, pages 73-87, 1978.

19. R. Paes Leme, V. Syrgkanis, and E. Tardos. The curse of simultaneity. In ITCS, 2012.

20. R. Paes Leme and E. Tardos. Pure and Bayes-Nash price of anarchy for generalized second price auction. In FOCS, 2010.

21. C. H. Papadimitriou, M. Schapira, and Y. Singer. On the hardness of being truthful. In FOCS, pages 250-259, 2008.

22. T. Roughgarden. Intrinsic robustness of the price of anarchy. In STOC '09, 2009.

23. T. Roughgarden. The price of anarchy in games of incomplete information. In Proceedings of the 13th ACM Conference on Electronic Commerce, EC '12, pages 862-879, New York, NY, USA, 2012. ACM.

24. M. Schapira and Y. Singer. Inapproximability of combinatorial public projects. In WINE, pages 351-361, 2008.

25. V. Syrgkanis. Bayesian games and the smoothness framework. CoRR, abs/1203.5155, 2012.

26. V. Syrgkanis and E. Tardos. Composable and efficient mechanisms. In STOC, 2013. 


\section{A Appendix}

\section{A.1 Omitted proofs from Section 5}

Theorem. For $k=1, n=2$ there exists an optimal equilibrium.

Proof. For $m=2$ it is easy to show existence of equilibrium by examining all possible cases. So we focus on the case $m \geq 3$.

Let $A=\operatorname{argmax}_{j} V_{j}$ be the optimal project. Also, let $B=\operatorname{argmax}_{j \neq A} v_{1 j}$ and $C=\operatorname{argmax}_{j \neq A, B} v_{2 j}$.

Suppose that $v_{1 A}-v_{1 C} \geq 0$ and $v_{2 A}-v_{2 B} \geq 0$. Then we claim that $b_{1 A}=$ $v_{1 A}-v_{1 C}, b_{2 A}=v_{2 A}-v_{2 B}, b_{1 B}=b_{2 C}=b_{1 A}+b_{2 A}=p$ and every other bid 0 is an equilibrium. Some useful properties of the above bidding: by the fact that $V_{A} \geq V_{B}, V_{C}$ and the definitions of $B, C$ we also get that: $b_{1 A} \geq v_{2 C}-v_{2 A} \geq$ $\max _{j \neq A, B} v_{2 j}-v_{2 A}$ and $b_{2 A} \geq v_{1 B}-v_{1 A} \geq \max _{j \neq A} v_{1 j}-v_{1 A}$.

Let's examine the deviations of agent 1 : He could include $B$ by slightly decreasing $b_{1 A}$ and slightly increasing $b_{1 B}$. Thus, we need: $v_{1 A}-b_{1 A} \geq v_{1 B}-p \Leftrightarrow$ $b_{2 A} \geq v_{1 B}-v_{1 A}$. He could include $C$ by slightly decreasing $b_{1 A}$ and slightly increasing $b_{1 C}$. Thus we need: $v_{1 A}-b_{1 A} \geq v_{1 C} \Leftrightarrow b_{1 A} \leq v_{1 A}-v_{1 C}$ which holds with equality. He could include some other item $j \neq B, C$ by placing a bid of $p$ on that: $v_{1 A}-b_{1 A} \geq v_{1 j}-p \Leftrightarrow b_{2 A} \geq v_{1 j}-v_{1 A}$. Similarly we can verify equilibrium for agent 2 .

Now we move to the case where some of $v_{1 A}-v_{1 C}$ or $v_{2 A}-v_{2 B}$ is negative. We observe that both of them cannot be negative: if they were then $V_{A}=$ $v_{1 A}+v_{2 A}<v_{1 C}+v_{2 B} \leq v_{1 B}+v_{2 B}=V_{B}$, which is a contradiction. Thus either $v_{1 A}<v_{1 C}$ and $v_{2 A} \geq v_{2 B}$ or $v_{1 A} \geq v_{1 C}$ and $v_{2 A}<v_{2 B}$. In the first case, since $V_{A} \geq V_{C}$ we have $v_{2 A} \geq v_{2 C}$. Thus $v_{2 A} \geq \max _{j \in[m]} v_{2 j}$. In the latter case, since $V_{A} \geq V_{C}$ we have $v_{1 A} \geq v_{1 B}$. Thus $v_{1 A} \geq \max _{j \in[m]} v_{1 j}$.

Thus, in both cases there is a agent whose maximum value item is the optimal item. Hence, we now construct the following equilibrium: Let $A=$ $\arg \max _{j \in M} v_{1 j}$ and $B=\arg \max _{j \in M} v_{2 j}$. Wlog assume that $v_{1 A}+v_{2 A} \geq v_{1 B}+$ $v_{2 B}$ and hence $A$ is the overall optimal item.

We claim the following is an equilibrium: Let $p \in\left[v_{2 B}-v_{2 A}, v_{1 A}-v_{1 A}\right]$. Since $v_{1 A}+v_{2 A} \geq v_{1 B}+v_{2 B} \Longrightarrow v_{1 A}-v_{1 B} \geq v_{2 B}-v_{2 A}$, we know that the above interval is not empty. Player 1 bids $p^{+}$for $A$ and 0 for everything else. Player 2 bids $p$ for $B$ and 0 for everything else. Project $A$ is selected.

Player 1 has two possible deviations. He could decrease his bid on $A$ and bid $p^{+}$on some other project $j$ to make it win. But by the definition of $A$, we know that $v_{1 A} \geq v_{1 j} \Longrightarrow v_{1 A}-p \geq v_{1 j}-p$. Hence, this is not profitable for him. Alternatively, he could just decrease his bid on $A$ and let $B$ win. His utility then would be $v_{1 B}$. But since $p \leq v_{1 A}-v_{1 B} \Longrightarrow v_{1 A}-p \geq v_{1 B}$. Hence, this is not profitable either.

Player 2 has also two possible deviations. He could slightly increase his bid on $B$ to make it win. But since $p \geq v_{2 B}-v_{2 A} \Longrightarrow v_{2 A} \geq v_{2 B}-p$. Alternatively he could bid $p^{+}$on some other project $j$. But by definition of $B$ we know: $v_{2 B} \geq v_{2 j}$. Combining with the region of $p$, we get: $p \geq v_{2 B}-v_{2 A} \geq v_{2 j}-v_{2 A} \Longrightarrow v_{2 A} \geq$ $v_{2 j}-p$. 
Theorem. Even for additive agents and $k=2$ there may not be a PNE.

Proof. We show that if $k=2$ and there is only one price setting project then there might not exist an equilibrium even for two agents with additive valuations. The instance constists of 2 agents, 3 projects $\{A, B, C\}$ and $k=2$. The valuations of the agents are:

$$
\left[v_{i j}\right]=\left[\begin{array}{lll}
1 & 2 & 3 \\
3 & 2 & 1
\end{array}\right]
$$

Since, two out of three projects are chosen then there will be only one price setting project. Any person that bids on the project that is excluded cannot possibly bid on the chosen projects at equilibrium since in that case he could just decrease his bid on the price setter and on the chosen project and strictly increase his utility. Thus at equilibrium some agent (payer) is bidding alone on the chosen projects and the other agent (price setter) is bidding only on the nonchosen project. Moreover, all the bids are equal to some $p$. We take all possible cases of chosen projects and payers and show that none can be an equilibrium:

- $A, B$ is chosen.

- 1 is the payer: Then it has to be: $p \leq v_{1 A}-v_{1 C} \Longrightarrow p \leq-2$

- 2 is the payer: Then it has to be: $v_{1 C}-v_{1 A} \leq p \leq v_{2 B}-v_{2 C} \Longrightarrow 2 \leq$ $p \leq 1$

$-B, C$ is chosen.

- 1 is the payer: Then it has to be: $v_{2 A}-v_{2 C} \leq p \leq v_{1 B}-v_{1 A} \Longrightarrow 2 \leq$ $p \leq 1$

- 2 is the payer: Then it has to be: $p \leq v_{2 C}-v_{2 A} \Longrightarrow p \leq-2$

$-A, C$ is chosen.

- 1 is the payer: Then it has to be: $p \leq v_{1 A}-v_{1 B} \Longrightarrow p \leq-1$

- 2 is the payer: Then it has to be: $p \leq v_{2 C}-v_{2 B} \Longrightarrow p \leq-1$

Theorem. It is NP-hard to compute a Pure Nash Equilibrium of the item bidding mechanism game for two agents with coverage valuations.

Proof. It is well known that given a single agent with a coverage valuation, finding the set $S$ that maximizes his value is NP-hard. Hence, we will just reduce the problem of finding the value maximizing set of a coverage valuation to finding an equilibrium of a two agent combinatorial public project auction.

Suppose that agent 1 has some valuation $v_{1}$ and agent 2 has a valuation $v_{2}$, such that $\forall S: v_{2}(S)=\epsilon v_{1}(S)$. Consider a Nash Equilibrium $b$ and with chosen set $S$. Let $P_{1}(S)$ be the total price paid by agent 1 and $P_{2}(S)$ the total price paid by agent 2 . agent 2 is paying at most $v_{2}(S)$. Each chosen project in the equilibrium receives the same amount of bids $p$. Hence, $P_{1}(S) \geq k p-v_{2}(S)=$ $k p-\epsilon v_{1}(S)$. agent 1 could potentially change the chosen set to any other set $S^{\prime}$ by bidding $p+\delta$ on $S^{\prime}$. Since that is not a profitable deviation:

$$
\begin{aligned}
v_{1}(S)-P_{1}(S) \geq v_{1}\left(S^{\prime}\right)-k p \Longrightarrow v_{1}(S)-k p+\epsilon v_{1}(S) & \geq v_{1}\left(S^{\prime}\right) \Longrightarrow \\
(1+\epsilon) v_{1}(S) & \geq v_{1}\left(S^{\prime}\right)
\end{aligned}
$$


Since $\epsilon$ can be arbitrarily small, this implies that $S \in \arg \max _{S^{\prime}} v_{1}\left(S^{\prime}\right)$. Hence, computing an equilibrium would imply computing the optimal set for the single agent optimization problem.

\section{A.2 Omitted Proofs from Section 6}

Theorem. For agents with arbitrary monotone valuations the Item-Bidding Mechanism is $\left(\frac{1}{2}, n \cdot k\right)$-smooth.

Proof. Consider a valuation profile $v$ and a bid profile $\boldsymbol{b}$. Let $O P T(v)$ be the optimal set of projects for valuation profile $v$. Let $p_{1}(\boldsymbol{b})$ be the total bid of the highest valued project under bid profile $\boldsymbol{b}$. Suppose that agent $i$ switches to $\boldsymbol{b}_{i}^{\prime}$ in which he draws a random bid $t$ uniformly at random from $\left[0, \frac{v_{i}(O P T(v))}{k}\right]$ (i.e. with density $\left.f(t)=\frac{k}{v_{i}(O P T(v))}\right)$ and submits this random bid $t$ on all the projects in $O P T(v)$. If $p_{1}(\boldsymbol{b})<t$ then the player gets all of the projects in $O P T(v)$ selected and hence gets a value of $v_{i}(O P T(v))$. The expected payment that he pays is at most his expected total bid, which is $k \frac{v_{i}(O P T(v))}{2 k}$. By the quasi-linearity of utilities and the linearity of expectation, his expected utility under this deviation is at least:

$$
\begin{aligned}
u_{i}\left(\boldsymbol{b}_{i}^{\prime}, \boldsymbol{b}_{-i}\right) & \geq \int_{p_{1}(\boldsymbol{b})}^{\frac{v_{i}(O P T(v))}{k}} v_{i}(O P T(v)) f(t) d t-\frac{v_{i}(O P T(v))}{2} \\
& =\int_{p_{1}(\boldsymbol{b})}^{\frac{v_{i}(O P T(v))}{k}} k \cdot d t-\frac{v_{i}(O P T(v))}{2} \\
& =\frac{v_{i}(O P T(v))}{2}-k \cdot p_{1}(\boldsymbol{b})
\end{aligned}
$$

By summing over all players and using the trivial fact that $p_{1}(\boldsymbol{b}) \leq \sum_{i} P_{i}(\boldsymbol{b})$ we get the theorem.

Theorem. For agents with fractionally subadditive monotone valuations the Item-Bidding Mechanism is $\left(\frac{1}{2}\left(1-\frac{1}{e}\right), n\right)$-smooth.

Proof. To simplify the notation in the proof we will assume that $k$, the number of chosen projects, is even. Consider a valuation profile $v$ and a bid profile $\boldsymbol{b}$. Let $O P T(v)$ be the optimal set of projects for valuation profile $v$. Let $\left(v_{i j}^{*}\right)_{j \in[m]}$ be the representative additive for player $i$ for set $O P T(v)$, i.e.

$$
v_{i}(O P T(v))=\sum_{j \in O P T(v)} v_{i j}^{*}=\max _{\ell \in L} \sum_{j \in O P T(v)} v_{i j}^{\ell}
$$

Assume that items $j$ are reorder such that projects 1 to $k / 2$ are the ones with the highest $v_{i j}^{*}$ in the above representative additive valuation. Hence, by definition, $\sum_{j=1}^{k / 2} v_{i j}^{*} \geq \frac{1}{2} v_{i}(O P T(v))$.

Suppose that agent $i$ switches to $\boldsymbol{b}_{i}^{\prime}$ in which for each project $j \in[1, \ldots, k / 2]$ he draws an independent random number $t_{j}$ with density $f_{j}\left(t_{j}\right)=\frac{1}{v_{i j}^{*}-t_{j}}$ and 
support $\left[0, v_{i j}^{*}\left(1-e^{-1}\right)\right]$ and submits this random bid $t_{j}$ on project $j \in O P T(v)$. He submits a 0 on any other project. Let $X(\boldsymbol{t}) \subseteq[\mathrm{m}]$ be the set of projects that are chosen for some random draw $\boldsymbol{t}=\left(t_{j}\right)_{j \in[m]}$ of the deviating bids of player $i$. Hence, a player's utility from the deviation is:

$$
u_{i}\left(\boldsymbol{b}_{i}^{\prime}, \boldsymbol{b}_{-i}\right)=\mathbb{E}_{\boldsymbol{t}}\left[v_{i}(X(\boldsymbol{t}))-\sum_{j \in X(\boldsymbol{t})} t_{j}\right]
$$

Using the fractionally subadditive property of the valuation we know that $v_{i}(X(\boldsymbol{t})) \geq$ $\sum_{j \in X(t)} v_{i j}^{*}$. Thus:

$$
u_{i}\left(\boldsymbol{b}_{i}^{\prime}, \boldsymbol{b}_{-i}\right) \geq \mathbb{E}_{t}\left[\sum_{j \in X(\boldsymbol{t})} v_{i j}^{*}-t_{j}\right]=\sum_{j \in[m]} \mathbb{E}_{t_{j}}\left[\left(v_{i j}^{*}-t_{j}\right) \cdot \mathbf{1}_{j \in X(\boldsymbol{t})}\right]
$$

Now, observe that for all $j \in[m], t_{j} \leq v_{i j}^{*}$, by the definition of the deviating bids. Thus, each term in the above sum is non-negative. Thus:

$$
u_{i}\left(\boldsymbol{b}_{i}^{\prime}, \boldsymbol{b}_{-i}\right) \geq \sum_{j=1}^{k / 2} \mathbb{E}_{t_{j}}\left[\left(v_{i j}^{*}-t_{j}\right) \cdot \mathbf{1}_{j \in X(\boldsymbol{t})}\right]
$$

Let $p_{t}(\boldsymbol{b})$ denote the $t$-th highest total bid under the initial bid profile $\boldsymbol{b}$. For any $j \in O P T(v)$, if $t_{j}>p_{k / 2+1}(\boldsymbol{b})$ then project $j$ is definitely selected, since player $i$ is bidding non-positive on only $k / 2$ projects and we know that the bids of the rest of the players exceed $p_{k / 2+1}$ on at most $k / 2$ projects. Thus we can lower bound each term in the above sum as follows:

$$
\begin{aligned}
u_{i}\left(\boldsymbol{b}_{i}^{\prime}, \boldsymbol{b}_{-i}\right) & \geq \sum_{j=1}^{k / 2} \mathbb{E}_{t_{j}}\left[\left(v_{i j}^{*}-t_{j}\right) \cdot \mathbf{1}_{t_{j}>p_{\frac{k}{2}+1}}(\boldsymbol{b})\right] \geq \sum_{j=1}^{k / 2} \int_{p_{\frac{k}{2}+1}(\boldsymbol{b})}^{\left(1-e^{-1}\right) v}\left(v_{i j}^{*}-t_{j}\right) f\left(t_{j}\right) d t_{j} \\
& =\left(1-\frac{1}{e}\right) \sum_{j=1}^{k / 2} v_{i j}^{*}-\sum_{j=1}^{k / 2} p_{\frac{k}{2}+1}(\boldsymbol{b}) \geq\left(1-\frac{1}{e}\right) \frac{1}{2} v_{i}(O P T(v))-\frac{k}{2} p_{\frac{k}{2}+1}(\boldsymbol{b}) \\
& \geq\left(1-\frac{1}{e}\right) \frac{1}{2} v_{i}(O P T(v))-\sum_{j=1}^{k / 2} p_{t}(\boldsymbol{b}) \geq\left(1-\frac{1}{e}\right) \frac{1}{2} v_{i}(O P T(v))-\sum_{i \in[n]} P_{i}(\boldsymbol{b})
\end{aligned}
$$

Summing over all agents we get the theorem.

Theorem. When bidder valuations are unit-demand then the Item-Bidding Mechanism is $\left(1-e^{-1}, \frac{n}{k}\right)$ semi-smooth.

Proof. Consider a valuation profile $v$ and a bid profile $\boldsymbol{b}$. Let $v_{i j}^{*}$ be the maximum valued project of each player: i.e. $v_{i j}^{*}=\max _{j \in[m]} v_{i j}$. Suppose that each player switches to the following randomized bid: he draws a random bid $t$ from distribution with density $f(t)=\frac{1}{v_{i j}^{*}-t}$ and support $\left[0,\left(1-e^{-1}\right) v_{i j}^{*}\right]$. Then he 
submits this random bid on project $j^{*}=\arg \max _{j \in[m]} v_{i j}$ and submits a 0 on all other projects. Let $p_{t}(\boldsymbol{b})$ be the total bid of the t-th highest valued project under the initial bid profile $\boldsymbol{b}$. For any $j \in O P T(v)$, if $t_{j}>p_{k}(\boldsymbol{b})$ then project $j$ is definitely selected. Thus a bidders utility from the deviation is at least:

$$
u_{i}\left(\boldsymbol{b}_{i}^{\prime}, \boldsymbol{b}_{-i}\right) \geq \int_{p_{k}(\boldsymbol{b})}^{\left(1-e^{-1}\right) v_{i j}^{*}}\left(v_{i j}^{*}-t\right) f(t) d t=\left(1-e^{-1}\right) v_{i j}^{*}-p_{k}(\boldsymbol{b})
$$

By summing up over all players and using the facts that $\sum_{i} v_{i j}^{*} \geq \sum_{i} v_{i}(O P T(v))$ and $p_{k}(\boldsymbol{b}) \leq \frac{1}{k} \sum_{t=1}^{k} p_{t}(\boldsymbol{b})=\frac{1}{k} \sum_{i} P_{i}(\boldsymbol{b})$ (by the definition of prices) we get the theorem. Observe that the deviation of the player depends only on his own valuation and hence the extra semi-smoothness property is satisfied. 